\title{
Structural Characterization of Anti-Complementary Polysaccharides from the Leaves of Artemisia princeps
}

\author{
Haruki Yamada ${ }^{1,3}$, Yasuo Otsuka ${ }^{1}$ and Satoshi Ōmura ${ }^{2}$
}

Received: January 7, 1986

\begin{abstract}
Structural characterizations of the anti-complementary acidic heteroglycans, AAF IIb-2 and IIb-3, obtained from the leaves of Artemisia princeps PAMP have been studied. AAF IIb-2 consists of rhamnose, xylose, arabinose, galactose, glucose and uronic acids (glucuronic acid and galacturonic acid) in the molar ratio of $7.6: 7.6: 13.0: 10.9: 3.0: 57.9$, and AAF IIb-3 consists of the same sugars in the ratio of $3.9: 2.6: 24.7: 19.7: 2.6: 46.5$. Methylation analysis including carboxyl-reduction and also selective enzymolysis using exo- $\alpha$-L-arabinofuranosidase suggested that AAF IIb-3 has a main chain consisting of $(1 \rightarrow 4)$-linked galacturonic acid and $(1 \rightarrow 2)$-linked rhamnose mostly substituted at the $0-4$ position. AAF IIb- 3 also contained arabino-3,6-galactan moiety and most of the arabinose was present as an $\alpha$-L-furanosyl residue in the non-reducing terminals and highly branched side chains which mostly attached to the $\mathrm{O}-3$ position of $(1 \rightarrow 6)$-linked galactopyranosyl residue. The basic structure of AAF IIb-2 is similar to that of AAF IIb-3, but IIb-3 has a higher arabinogalactan content than IIb-2.
\end{abstract}

\section{List of Abbreviations}

$\mathrm{C}_{5} \mathrm{H}_{5} \mathrm{~N} \quad$ : pyridine

THF : tetrahydrofuran

TFA : trifluoroacetic acid

${ }^{1} \mathrm{H}-\mathrm{NMR}$ : proton magnetic resonance

$\mathrm{NaBH}_{4}$ : sodium borohydride

$\mathrm{NaBD}_{4}$ : sodium borodeuteride

Araf : arabinofuranose

Gal : galactopyranose

Ara : arabinopyranose

Xyl : xylopyranose

Rha : rhamnopyranose

GalA : galacturonic acid

\section{Introduction}

Artemisiae Argyi Folium, the leaves of $A$. princeps Pamp (Japanese name $=$ Gaiyō) is a well known Chinese crude drug clinically used in the treatment of colic pain, vomiting and diarrhea, and irregular bleeding from uterus.

Previously, we have reported on the purification and chemical properties of anti-complementary polysaccharides, AAF IIb-2 and IIb-3 from the leaves of $A$. princeps Pamp (1). AAF IIb-2 and IIb-3 are suggested to be structurally related acidic heteroglycans because both polysaccharides were composed of the same component sugars but in different molar ratios (1). The structural characterizations of AAF IIb-2 and IIb-3 are studied in the present paper.

\footnotetext{
1 Oriental Medicine Research Center of the Kitasato Institute, 5-9-1, Shirokane, Minato-ku, Tokyo 108, Japan.

2 The Kitasato Institute, 5-9-1, Shirokane, Minato-ku, Tokyo 108, Japan,

${ }^{3}$ Address for correspondence.
}

\section{Materials and Methods}

\section{Materials}

The leaves of Artemisia princeps Pamp (Japanese name = Gaiyō) were purchased from Uchida Wakanyaku Co. Ltd., Tokyo, Japan. $\alpha$-LArabinofuranosidase from Rhodotorula flava (2) was a kind gift from Dr. Naoto Shibuya (National Food Research Institute, Ministry of Agriculture, Forestly and Fisheries, Japan). Bio-gel P-2 (200-400 mesh) was obtained from Bio-Rad. Sephadex LH-20 was purchased from Pharmacia and Sep-Pak C18 cartridge from Waters Associates Inc. Spectra Pore 6 was from SPECTRUM Medical Industries Inc.

\section{General methods}

The total carbohydrate and uronic acid contents were determined by the phenol-sulfuric acid method (3) and $m$-hydroxydiphenyl method (4), respectively, using arabinose and galacturonic acid as the respective standards. TLC was performed on cellulose-coated plastic sheet (Merck) with EtOAc- $\mathrm{C}_{5} \mathrm{H}_{5} \mathrm{~N}-\mathrm{HOAc}-\mathrm{H}_{2} \mathrm{O}(5: 5: 1: 3)$ as the solvent system. Reducing sugars were detected with alkaline silver nitrate (5), and uronic acid with $p$-anisidine hydrochloride (6). GLC (Shimadzu GC-6A) was equipped with a flame ionization detector (FID) and a glass column $(0.3 \times 200 \mathrm{~cm})$ of $3 \%$ ECNSS-M on Uniport HP at $180^{\circ} \mathrm{C}$ for component sugar analysis. Nitrogen was used as carrier gas at flow rate of $60 \mathrm{ml} / \mathrm{min}$. Polysaccharide $(0.1-1 \mathrm{mg}$ ) was hydrolyzed with $2 \mathrm{M}$ TFA at $121^{\circ} \mathrm{C}$ for $1.5 \mathrm{~h}$. The hydrolyzate containing neutral sugar and uronic acid was converted to alditol acetates by the method of Jones and Albersheim (7). The hydrolyzates were reduced to alditol and aldonic acid by the treatment with $\mathrm{NaBH}_{4}$ in $1 \mathrm{~N} \mathrm{NH}_{4} \mathrm{OH}$. The aldonic

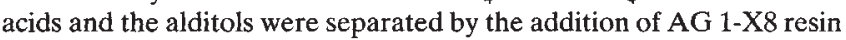
$\left(\mathrm{OAc}^{\Theta}\right.$ form). Unbound alditols were then acetylated with $\mathrm{Ac}_{2} \mathrm{O}$ at $121^{\circ} \mathrm{C}$ for $3 \mathrm{~h}$. The aldonic acids were eluted from the resin with $1 \mathrm{~N}$ $\mathrm{HC} 1$, and the $\mathrm{HCl}$ eluate was evaporated to dryness at $40^{\circ} \mathrm{C}$. converting the aldonic acid to aldonolactones. The aldonolactones were reduced with $\mathrm{NaBH}_{4}$ to the corresponding alditols, dried and acetylated. Alditol acetates were analyzed by GLC (8).

Purification of anti-complementary polysaccharides, AAF IIb-2 and $I I b-3$

The crude polysaccharide from the leaves of $A$. princeps Pamp was prepared by hot-water extraction and dialysis (1). The crude polysaccharide was purified by ion exchange chromatography on DEAESepharose, affinity chromatography on Ricinus communis-agglutinin conjugated Sepharose and gel filtrations on Sephadex G-100 and Sepharose CL-4B as reported previoulsy (1).

\section{Methylation analysis of polysaccharides}

Before per- $O$-methylation, AAF IIb-2 and AAF IIb-3 were passed through the column of $\mathrm{AG} 50 \mathrm{~W}-\mathrm{X} 8 \mathrm{resin}\left(\mathrm{H}^{+}\right.$form) in order convert all of the carboxyl groups into protonated form because this treatment permits more complete $O$-methylation of uronic acid containing polysaccharides (9). Then AAF IIb-2 and IIb- $3(2 \mathrm{mg})$ were methylated by the Hakomori procedure (10) and the completeness of the methylation was checked by using triphenylmethane (11). The per- $O$-methylated polysaccharides were purified by gel filtration on Sephadex LH20 (12) or reversed-phase chromatography on Sep-Pak C18 cartridge (9). Per- $O$-methylated polysaccharides were eluted from the Sep-Pak C18 cartridge with $100 \% \mathrm{CH}_{3} \mathrm{CN}$, followed by $100 \% \mathrm{EtOH}$ as described by Waeghe et al. (9). 
Table I. Methylation analysis of AAF IIb-2 and IIb-3.

\begin{tabular}{|c|c|c|c|c|c|}
\hline \multirow{2}{*}{$\begin{array}{l}\text { Methylated alditol } \\
\text { acetate derivatives }\end{array}$} & \multirow{2}{*}{$\mathrm{T}^{\mathrm{a}}$} & \multicolumn{2}{|c|}{ Mol \% } & \multirow{2}{*}{$\begin{array}{l}\text { Major mass spectral } \\
\text { fragments }(m / z)^{c}\end{array}$} & \multirow{2}{*}{ Linkage } \\
\hline & & IIb-2 & $\mathrm{IIb}-3$ & & \\
\hline $1,4-\mathrm{Ac}_{2}-2,3,5-\mathrm{Me}_{3}$-arabinitol & 0.44 & 11.8 & 19.2 & $43,45,71,87,101,117,129,161$ & Araf $\rightarrow$ \\
\hline $1,5-\mathrm{Ac}_{2}-2,3,4-\mathrm{Me}_{3}$-xylitol & 0.58 & 4.0 & 1.3 & $43,71,87,101,117,161$ & $\mathrm{Xyl} l^{1} \rightarrow$ \\
\hline $1,2,5-\mathrm{Ac}_{3}-3,4-\mathrm{Me}_{2}$-rhamnitol & 0.87 & 8.3 & 3.3 & $43,87,89,99,129,131,189$ & $\rightarrow{ }^{2} \mathrm{Rha}^{1} \rightarrow$ \\
\hline $1,4,5-\mathrm{Ac}_{3}-2,3-\mathrm{Me}_{2}$-arabinitol & 1.05 & 13.4 & 17.3 & $43,87,101,117,129,189$ & $\rightarrow{ }^{5}$ Araf ${ }^{4} \rightarrow$ or $\rightarrow{ }^{4} \mathrm{Ara}^{1} \rightarrow$ \\
\hline $1,5-\mathrm{Ac}_{2}-2,3,4,6-\mathrm{Me}_{4}$-galactitol & 1.18 & 7.9 & 6.9 & $\begin{array}{l}43,45,71,87,101,117,129,145 \\
161,205\end{array}$ & $\mathrm{Gal}^{\mathrm{I}} \rightarrow$ \\
\hline $1,2,4,5-\mathrm{Ac}_{4}-3$-Me-rhamnitol & 1.65 & 10.3 & 3.3 & $43,87,101,129,143,189,203$ & $\rightarrow{ }_{4}^{2} \mathrm{Rha}^{1} \rightarrow$ \\
\hline $1,3,4,5-\mathrm{Ac}_{4}-2-\mathrm{Me}$-arabinitol & 1.79 & 15.7 & 11.7 & $45,85,117,201,261$ & $\rightarrow{ }_{5}^{3} \mathrm{Araf} \rightarrow$ \\
\hline $1,3,5-\mathrm{Ac}_{3}-2,4,6-\mathrm{Me}_{3}$-galactitol & 1.96 & 3.6 & 4.4 & $\begin{array}{l}43,45,87,101,113,117,129 \\
131,161,233\end{array}$ & $\rightarrow{ }^{3} \mathrm{Gal} \rightarrow$ \\
\hline $1,4,5-\mathrm{Ac}_{3}-2,3,6-\mathrm{Me}_{3}$-galactitol & 2.15 & 14.3 & 11.1 & $43,45,87,101,117,129,161,233$ & $\rightarrow{ }^{4} \mathrm{Gal}^{1} \rightarrow$ \\
\hline $1,5,6-\mathrm{Ac}_{3}-2,3,4-\mathrm{Me}_{3}$-galactitol & 2.82 & 4.7 & 8.7 & $43,87,99,101,117,129,161,189$ & $\rightarrow{ }^{6} \mathrm{Gal}^{1} \rightarrow$ \\
\hline $1,4,5,6-\mathrm{Ac}_{4}-2,3-\mathrm{Me}_{2}$-galactitol & 4.55 & 4.2 & 0.6 & $43,85,87,99,101,117,127,261$ & $\rightarrow{ }_{6}^{4} \mathrm{Gal}^{1} \rightarrow$ \\
\hline $1,3,5,6-\mathrm{Ac}_{4}-2,4-\mathrm{Me}_{2}$-galactitol & 4.95 & 1.8 & 12.8 & $43.87,117,129,139,159,189,233$ & $\rightarrow_{6}^{3} \mathrm{Gal}^{1} \rightarrow$ \\
\hline
\end{tabular}

a Retention times of the corresponding alditol acetates relative to that of $1,5-\mathrm{Ac}_{2}-2,3,4,6-\mathrm{Me}_{4}$ glucitol on an OV-225 column.

b Calculated from peak areas and mol masses of derivatives.

c Assigned in the literature (14).

The fully methylated polysaccharides were reduced with $\mathrm{NaBD}_{4}$ in $27: 73(\mathrm{v} / \mathrm{v})$ 95\% EtOH-THF (13). Methyl-esterified hexosyluronic acid residues reduced by this procedure were converted into the corresponding 6,6-dideuteriolabeled hexosyl residue (9). Per- $O$-methylated and reduced polysaccharides were hydrolyzed with $2 \mathrm{M} \mathrm{TFA}$ at $121^{\circ} \mathrm{C}$ for $1 \mathrm{~h}$. The hydrolyzates were reduced to the corresponding partially $O$-methylated alditols with $\mathrm{NaBH}_{4}$ in $1 \mathrm{M}$ ammonia in $95 \% \mathrm{EtOH}$. The resulting partially $O$-methylated alditols were acetylated with $\mathrm{Ac}_{2} \mathrm{O}$ at $121^{\circ} \mathrm{C}$ for $3 \mathrm{~h}$, and then alditol acetate derivatives were analyzed by GLC on a Hewlett Packard 5840 A gas chromatograph equipped with dual flame ionization detectors. Samples were injected into a DB-1 capillary column $(30 \times 0.25 \mathrm{~mm}$ i.d., J and W Scientific Inc. U.S.A.) with splitless injection. The flow rate of the carrier gas, helium, was 0.9 $\mathrm{ml} / \mathrm{min}$. The gas chromatograph was programmed at $150^{\circ} \mathrm{C}$ for $1 \mathrm{~min}$, followed by a rate of $2^{\circ} \mathrm{C} / \mathrm{min}$ to $210^{\circ} \mathrm{C}$. All GLC flame ionization responses to partially methylated alditol acetates were corrected to mol responses as described by Sweet et al. (14). Partially methylated alditol acetates were also analyzed by GLC-MS (15). GLC-MS was performed on a Hitachi M-80 instrument equipped with OV-1 fused silica capillary column ( $25 \mathrm{~m} \times 0.25 \mathrm{~mm}$ i.d., Gaskuro Kogyo Inc., Tokyo) at 145 to $205^{\circ} \mathrm{C}\left(1^{\circ} \mathrm{C} / \mathrm{min}\right)$ with a split ratio of $80: 1$ and operated at an ionization voltage of $20 \mathrm{eV}$ with an ion source temperature at $180^{\circ} \mathrm{C}$. Glass column of $1 \%$ OV 225 on Uniport HP was also used for GLC and GLCMS at $170^{\circ} \mathrm{C}$.

\section{Exo- $\alpha-\mathrm{L}-$-arabinofuranosidase digestion of polysaccharides}

AAF IIb- 3 was digested with $\alpha$-L-arabinofuranosidase from $R$. flava as reported previously (16). The enzyme digested polysaccharide (yield, $75.4 \%$ ) was recovered in the void volume by gel filtration on a column $(2.0 \times 52 \mathrm{~cm})$ of Bio-gel P-2 at $55^{\circ} \mathrm{C}$ or in the non-dialyzable portion after dialysis against water using Spectra pore 6 (molecular weight cut off $<1000$ ). The fraction eluted in the included volume or the dialyzable portion was desalted with $\mathrm{AG} 50 \mathrm{~W} \mathrm{X} 8\left(\mathrm{H}^{+}\right.$form $)$.

\section{Reduction of carboxyl groups in polysaccharides}

The carboxyl groups of uronic acid in the polysaccharides were reduced with 1-ethyl-3-(dimethylaminopropyl)-carbodiimide and $\mathrm{NaBH}_{4}$ according to the method of Taylor and Conrad (17), and the reaction mixture was dialyzed and lyophilized.

\section{NMR studies}

${ }^{1} \mathrm{H}$-NMR spectra measured in $\mathrm{D}_{2} \mathrm{O}$ at $400 \mathrm{MHz}$ at $80^{\circ} \mathrm{C}$, using Varian $\mathrm{XL}-400$. Chemical shifts $(\delta)$ were expressed in ppm relative to that of sodium 3-(trimethylsilyl)-propane-1-sulfonate (TSP).

\section{Results}

AAF IIb-2 and IIb-3 were composed of rhamnose, xylose, arabinose, galactose, glucose, galacturonic acid, and glucuronic acid in the molar ratios of $7.6: 7.6: 13.0: 10.9: 3.0$ : $51.2: 6.7$ and $3.9: 2.6: 24.7: 19.7: 2.6: 15.0: 31.5$, respectively. AAF IIb- 2 and IIb-3 showed positive specific rotations $\left([\alpha]_{D}:+114.3^{\circ}\right.$ and $\left.+67.0^{\circ}\right)$. The ${ }^{1} \mathrm{H}-\mathrm{NMR}$ spectrum of major anti-complementary polysaccharide, AAF IIb-3, showed signals due to the methyl protons of the rhamnosyl residue at $\delta=$ $1.25, O$-acetyl protons at $\delta=2.0$ and 2.13, $O$-methyl protons as carboxylic acid methyl esters at $\delta=3.80$, and anomeric protons due to $\beta$ - and $\alpha$-configurations at $\delta=4.5-4.8$ and 4.9-5.3. AAF IIb-2 and IIb-3 were methylated by the methylsulphinylcarbanion and methyl iodide in dimethyl sulfoxide, followed by the reduction with $\mathrm{NaBD}_{4}$. The fully methylated and reduced polysaccharides were hydrolyzed with acid, and the products were analyzed as the alditol acetates by GLC and GLC-MS. The results suggest that both polysaccharides contained arabinofuranosyl residues as non-reducing end groups, $(1 \rightarrow 5)$ linked arabinofuranosyl or $(1 \rightarrow 4)$-linked arabinopyranosyl residues and $(1 \rightarrow 3,5)$-linked arabinofuranosyl residues, and galactopyranosyl residues as non-reducing end groups and $(1 \rightarrow 3)$-, $(1 \rightarrow 4)-,(1 \rightarrow 6)$-, and $(1 \rightarrow 3,6)$-linked galactopyranosyl residues, xylopyranosyl residues as non-reducing end groups, and $(1 \rightarrow 2)$ - and $(1 \rightarrow 2,4)$-linked rhamnopyranosyl residues in the chain (Table I). The identification of 2,3-di- $O$-methyl-1, 4 , 5, 6-tetra- $O$-acetylgalactitol labelled with deuterium at the O-6 position in the methylated and reduced polysaccharides confirmed that the compound was derived from the $(1 \rightarrow 4)$-linked galacturonic acid residue in the original polysaccharides. To confirm the structure of uronic acid moiety of AAF IIb-3, carboxyl reduced polysaccharide was also subjected to methylation analysis. The presence of glucuronic acid as non-reducing end groups and $(1 \rightarrow 4)$-linked galacturonic acid are suggested by the increase of 2, 3, 4, 6-tetra- $O$-methylglucose and 2, 3, 6-tri- $O$ methylgalactose in the carboxyl-reduced AAF IIb-3 (Table II). The yield of the reduction product, 2,3-di- $O$-methylgalactose, derived from fully methylated and reduced AAF IIb-3 was very low value in comparison with expected value based on the uronic acid content of the original polysaccharide. It was 
suggested that the low recovery of the reduction product may be caused by the low efficiency of the reduction step and the loss of uronic acid residues during Hakomori-methylation by $\beta$ elimination. To obtain more detailed information about the structure of the side chains of AAF IIb-3, AAF IIb-3 was digested with exo- $\alpha$-L-arabinofuranosidase from $R$. flava. Then the reaction products were fractionated on a column of Bio-gel P-2 (data not shown), and two carbohydrate fractions that were eluted in the void and retained volumes were obtained. Arabinose was the only sugar detected in the retained volume by TLC and GLC. The material recovered in the void volume consisted of rhamnose, arabinose, xylose, galactose, and glucose in a molar ratio of $0.35: 0.28: 0.16: 1.0: 0.1$ as the neutral sugars. These results lead to the conclusion that most of arabinosyl residues in AAF IIb- 3 were linked in an $\alpha$-configuration in the furanose form. The ${ }^{1} \mathrm{H}-\mathrm{NMR}$ spectrum of exo- $\alpha$ L-arabinofuranosidase digested AAF IIb-3 showed that the signal at $\delta=2.0$ had decreased in intensity, suggesting that a part of $O$-acetyl group may be linked to a part of arabinofuranosyl residues. The results of methylation analysis of the arabinofuranosidase digested AAF IIb-3 showed that the molar ratio of the terminal, $(1 \rightarrow 5)-$, and $(1 \rightarrow 3,5)$-linked arabinofuranosyl residues, and $(1 \rightarrow 3)$ - and $(1 \rightarrow 3,6)$-linked galactosyl residues had decreased significantly, and that the molar ratio of the terminal and $(1 \rightarrow 6)$-linked galactosyl residues had increased compared to AAF II-3 (Table II). When AAF IIb-2 was digested with the same enzyme, similar results with AAF IIb-3 were obtained, but further studies of AAF IIb2 were not possible because of the small quantity available.

\section{Discussion}

The major anti-complementary polysaccharide, AAF IIb-3, was mainly composed of arabinose and galactose while the minor one, AAF IIb-2, was mainly composed of rhamnose, xylose, arabinose, and galactose (1). Previously, a significant amount of galacturonic acid was detected in these heteroglycans by TLC of the acid hydrolyzates (1), but in the present paper, glucuronic acid was also identified by GLC analysis. AAF IIb-2 contained more substantial amounts of $(1 \rightarrow 4)$ linked galacturonic acid and $(1 \rightarrow 2)$-linked rhamnopyranosyl residues than AAF IIb-3. These results indicate that AAF IIb-2
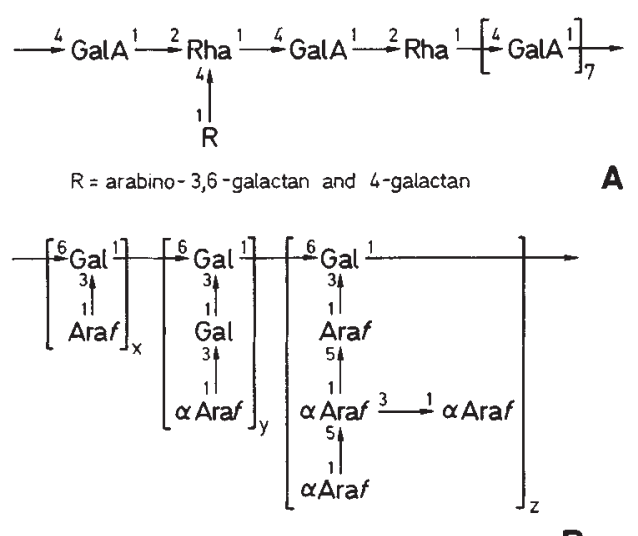

$\mathbf{B}$

Fig. 1. A: A possible partial structural fragment of AAF IIb-2 and IIb-3. Xylosyl and glucuronic acid residues are present at non-reducing terminals somewhere in the polysaccharides.

B: A possible structural fragment of the arabino-3,6-galactan moiety in AAF IIb-2 and IIb-3.

and IIb-3 have a rhamnogalacturonan main chain as shown in pectic polysaccharides similar to those isolated from cotyledon meals and hulls of Glycine $\max (18,19)$, leaves and stems of Medicago sativa (20), suspension cultured sycamore cell walls (21), rice endosperm cell walls (22), the fruits of Zizyphus jujuba Millar var. inermis Rehd (23) and suspension-culture cells and leaves of Nicotiana tabacum $(24,25)$. Darvill et al. classified that there are two types of rhamnogalacturonan I and II (26). AAF IIb-2 and IIb-3 apeared to be rhamnogalacturonan I type polysaccharides because apiose, 2-methylfucose, and 2-methylxylose were not detected in these acidic heteroglycans. The results of methylation analysis of AAF IIb-3 before and after $\alpha$-L-arabinofuranosidase digestion, suggest that the highly branched arabinogalactan moiety is a $(1 \rightarrow 3)$ - and $(1 \rightarrow 6)$-linked galactan, possessing attached highly branched arabinofuranosyl side chains at O-3 of some of the $(1 \rightarrow 6)$ linked galactosyl residues in the main chain or the interior chains of galactose residues mutually joined by $(1 \rightarrow 3)$ - and $(1 \rightarrow 6)$-linkages. This structure moiety was very similar to the anti-complementary arabino-3,6-galactans from the roots of

Table II. Methylation analysis of AAF IIb-3, carboxyl-reduced IIb-3 and IIb-3 $\alpha$-L-arabinofuranosidase ( $\alpha$-L-Arafase) digest

\begin{tabular}{|c|c|c|c|c|}
\hline \multirow{2}{*}{$\begin{array}{l}\text { Methylated alditol } \\
\text { acetate derivatives }\end{array}$} & \multicolumn{4}{|c|}{ Mol \% } \\
\hline & IIb-3* & Reduced IIb-3 & $\alpha$-L-Arafase treated & Linkage \\
\hline 1,4-Ac $-2,3,5-\mathrm{Me}_{3}$-arabinitol & 19.2 & 8.7 & $6.7(5.1)^{\mathrm{a}} \downarrow$ & Araf $\rightarrow$ \\
\hline 1,5- $\mathrm{Ac}_{2}-2,3,4-\mathrm{Me}_{3}$-xylitol & 1.6 & 6.2 & $2.1(1.6)$ & $\mathrm{Xyl}^{1} \rightarrow$ \\
\hline $1,2,5-\mathrm{Ac}_{3}-3,4-\mathrm{Me}_{2}$-rhamnitol & 4.1 & 3.0 & $6.9(5.2)$ & $\rightarrow{ }^{2} \mathrm{Rha}^{1} \rightarrow$ \\
\hline $1,5-\mathrm{Ac}_{2}-2,3,4,6-\mathrm{Me}_{4}$-glucitol & - & $6.2 \uparrow$ & $-(-)$ & $\mathrm{Glc}^{1} \rightarrow$ \\
\hline $1,4,5-\mathrm{Ac}_{3}-2,3-\mathrm{Me}_{2}$-arabinitol & 16.0 & 7.0 & $10.2(7.7) \downarrow$ & $\rightarrow^{5} \mathrm{Ara}^{4} \rightarrow$ or $\rightarrow^{4} \mathrm{Ara}^{1} \rightarrow$ \\
\hline $1,5-\mathrm{Ac}_{2}-2,3,4,6-\mathrm{Me}_{4}$-galactitol & 15.1 & 12.9 & $25.6(19.5) \uparrow$ & $\mathrm{Gal}^{1} \rightarrow$ \\
\hline $1,2,4,5-\mathrm{Ac}_{4}-3-\mathrm{Me}$-rhamnitol & 4.0 & 1.1 & $6.4(4.8)$ & $\rightarrow_{4}^{2} \mathrm{Rha}^{1} \rightarrow$ \\
\hline $1,3,4,5-\mathrm{Ac}_{4}-2-\mathrm{Me}$-arabinitol & 14.6 & 4.2 & $3.6(2.7) \downarrow$ & $\rightarrow 5^{3} \mathrm{Araf} \rightarrow$ \\
\hline $1,3,5-\mathrm{Ac}_{3}-2,4,6-\mathrm{Me}_{3}$-galactitol & 7.4 & 3.8 & $9.2(7.0)$ & $\rightarrow{ }^{3} \mathrm{Gal}^{1} \rightarrow$ \\
\hline $1,4,5-\mathrm{Ac}_{3}-2,3,6-\mathrm{Me}_{3}$-galactitol & 8.2 & $42.7 \uparrow$ & $11.2(8.5)$ & $\rightarrow^{4} \mathrm{Gal}^{1} \rightarrow$ \\
\hline $1,5,6-\mathrm{Ac}_{3}-2,3,4-\mathrm{Me}_{3}$-galactitol & 4.6 & 2.3 & $12.6(9.6) \uparrow$ & $\rightarrow{ }^{6} \mathrm{Gal}^{1} \rightarrow$ \\
\hline $1,3,5,6-\mathrm{Ac}_{4}-2,4-\mathrm{Me}_{2}$-galactitol & 5.2 & 1.9 & $5.4(3.6) \downarrow$ & $\underset{\rightarrow 6}{\rightarrow 3} \mathrm{Gal}^{1} \rightarrow$ \\
\hline
\end{tabular}

* Methylated different IIb-3 preparation from Table I.

${ }^{\text {a }}$ Molar ratio in parethesis culculated relative to the molar ratio of 1, 5- $\mathrm{Ac}_{2}-2,3,4-\mathrm{Me}_{3}$-xylitol in IIb-3.

$\downarrow$ decreased, $\uparrow$ increased. 
Angelica acutiloba Kitagawa (AR-arabinogalactan IIa and IIb1) $(16,27,28)$ and the arabinogalactans from Zea shoots (29).

When AAF IIb-2 and IIb-3 were digested with exo- $\alpha-\mathrm{L}-$ arabinofuranosidase, a significant amount of the arabinosyl residue still remained in the enzyme resistant polysaccharide. The results of methylation analysis of the enzyme digested AAF IIb-3 indicate that the terminal and $(1 \rightarrow 5)$-linked arabinofuranosyl residue had been retained significantly compared to AAF IIb-3. These results suggest that arabinogalactan moiety of AAF IIb-3 contained a highly branched arabinan moiety, possessing attached arabinofuranosyl side chains at $\mathrm{O}$ 3 of some of the $(1 \rightarrow 5)$-linked arabinofuransyl residues (Fig. 1 ), and the enzyme may be cleaved only at outer side chains of the branching point. The structure of this branched arabinan moiety was also similar to that of AR-arabinogalactan IIb-1 (28) from A. actiloba. The rhamnogalacturonan moieties in AAF IIb-2 and IIb-3 may be present in the inner core region because when AAF IIb-3 was subjected to partial acid hydrolysis, galacturonic acid, rhamnose, and galactose were detected as major component sugars in the acid resistant polysaccharide (unpublished results). This result also suggested that the arabinogalactan side chain may be attached to the rhamnogalacturonan backbone through position 4 of $(1 \rightarrow 2)$-linked rhamnose residue in AAF IIb-2 and IIb-3 (Fig. 1). AAF IIb-2 was shown to have more potent anti-complementary activity than IIb-3 (1), but both contained similar glycosidic linkages with different molar ratios.

Although rhamnogalacturonan moieties and non-reducing end groups of xylosyl and glucuonic acid residues were more predominant than the arabinogalactan moiety in AAF IIb-2, the elucidation of the structural difference between AAF IIb-2 and IIb-3 in association with the activity must await further detailed analysis.

\section{Acknowledgements}

A part of this work was supported by the fund of Tsumura-Juntendo Co. Ltd., Tokyo. We are grateful to H. Kiyohara and N. Tsuji for their technical assistance. We are grateful to Dr. Jyong-Chol Cyong of this Institute and Prof. Y. Ueno of Tokyo Science University for his encouragement through this research.

\section{References}

(1) Yamada, H., Ohtani, K., Kiyohara, H., Cyong, J-C., Otsuka, Y., Ueno, Y., Ōmura, S. (1985) Planta Med. 121.

(2) Uesaka, E., Sato, M Raiju, M., Kaji, A. (1978) J. Bacterial. $133,1073$.

(3) Dubois, M., Gilles, K. A., Hamilton, J. K., Rebers, P. A., Smith, F. (1956) Anal. Chem. 28, 350.

(4) Blumenkranz, N., Asboe-hansen, G. (1973) Anal. Biochem. 54, 484.

(5) Trevelyan, W. E., Procter, D. P., Harrison, J. S. (1950) Nature (London) 166, 444

(6) Hough, L., Jones, J. K. N., Wadman, W. H. (1950) J. Chem. Soc. 1702.

(7) Jones, T. M., Albersheim, P. (1972) Plant Physiol. 49, 926.

(8) Yamada, H., Kiyohara, H., Otsuka, Y. (1984) Phytochemistry $23,587$.

(9) Waeghe, T. J., Darvill, A. G., McNeil, M., Albersheim, P. (1983) Carbohydr. Res. 123, 281.

(10) Hakomori, S. (1964) J. Biochem. (Tokyo) 55, 205.

(11) Rauvala, H. (1979) Carbohydr. Res. 72, 257.

(12) Stellner, K., Saito, H., Hakomori, S. (1973) Archs. Biochem. Biophys. 155, 464.

(13) Dutton, G. G. S., Mackie, K. L., Savage, V., Stephenson, M. D. (1978) Carbohydr. Res. 66, 125.

(14) Sweet, D. P., Shapiro, R. H., Albersheim, P. (1975) Carbohydr. Res. 40, 217.

(15) Bjorndal, H., Lindberg, B., Svensson, S. (1967) Carbohydr. Res. $5,433$.

(16) Yamada, H., Kiyohara, H., Cyong, J-C., Otsuka, Y (1985) Molec. Immunol. 22, 295.

(17) Taylor, R. L., Conrad, H. E. (1972) Biochemistry 11, 1383.

(18) Aspinal, G. O., Cottrell, I. W., Egan, S. V., Morrison, I. M., Whyte, J. N. C. (1967) J. Chem. Soc. 1071.

(19) Aspinal, G. O., Hunt, K., Morrison, I. M. (1967) J. Chem. Soc. 1080.

(20) Aspinal, G. O., Gestetner, B., Molloy, J. A., Uddin, M. (1968) J. Chem. Soc. 2554.

(21) McNeil, M., Darvill, A. G., Albershiim, P., (1980) Plant Physiol. $66,1128$.

(22) Shibuya, N., Nakane, R. (1984) Phytochemistry 23, 1425.

(23) Shimizu, N., Tomoda, M. (1983) Chem. Pharm. Bull. 31, 499.

(24) Kato, K., Noguchi, M. (1976) Agric. Biol. Chem. 40, 1923.

(25) Eda, S., Kato, K. (1980) Agric. Biol. Chem. 44, 2793.

(26) Darvill, A., McNeil, M., Albersheim, P., Delmer, D. P. (1980) in The Biochemistry of Plants (Tolbert N. E., ed.) vol. 1/91 Academic Press, New York.

(27) Yamada, H., Kiyohara, H., Cyong, J-C., Kojima, Y., Kumazawa, Y., Otsuka, Y. (1984) Planta Med. 50, 163.

(28) Kiyohara, H., Yamada, H., Cyong, J-C., Otsuka, Y. J. Pharmacobio, Dyn. in press.

(29) Kato, Y., Nevins, D. J. (1984) Plant Physiol. 74, 562. 